\title{
Sequential Docetaxel in $\geq 7$ Cycles Followed by Cabazitaxel Improves Oncological Outcomes in Patients with Metastatic Castration-Resistant Prostate Cancer
}

\author{
Seiichi Kato, ${ }^{1}$ Manabu Takai, ${ }^{2}$ Koji Iinuma, ${ }^{2}$ Shota Fujimoto, ${ }^{3}$ Masahiro Nakano, ${ }^{4}$ \\ Takashi Ishida, ${ }^{1}$ Masahiro Uno, ${ }^{1}$ Masayoshi Tamaki, ${ }^{3}$ Mitsuhiro Taniguchi, ${ }^{4}$ \\ Hisao Komeda, ${ }^{3}$ Yoshito Takahashi, ${ }^{4}$ and Takuya Koie ${ }^{2}{ }^{2}$ \\ ${ }^{1}$ Department of Urology, Ogaki Municipal Hospital, 4-86 Minaminokawacho, Ogaki 503-8502, Japan \\ ${ }^{2}$ Department of Urology, Gifu University Graduate School of Medicine, 1-1 Yanagido, Gifu 501-1194, Japan \\ ${ }^{3}$ Department of Urology, Gifu Municipal Hospital, 7-1 Kashimacho, Gifu 500-8513, Japan \\ ${ }^{4}$ Department of Urology, Gifu Prefectural General Medical Center, 4-6-1 Noishiki, Gifu 500-8717, Japan
}

Correspondence should be addressed to Takuya Koie; goodwin@gifu-u.ac.jp

Received 25 September 2020; Revised 27 December 2020; Accepted 4 January 2021; Published 12 January 2021

Academic Editor: Tomasz Brzozowski

Copyright (C) 2021 Seiichi Kato et al. This is an open access article distributed under the Creative Commons Attribution License, which permits unrestricted use, distribution, and reproduction in any medium, provided the original work is properly cited.

Background. Docetaxel (DOC) was the first regimen that increased the survival and became the standard-of-care in patients with metastatic castration-resistant prostate cancer (mCRPC). However, it is unclear whether switching to second-line chemotherapy or optimal sequencing of cabazitaxel (CBZ) ensures better clinical outcomes. We aimed to evaluate the efficacy of sequential therapy with DOC and CBZ and the effect of the number of prior DOC cycles on oncological outcomes in patients with mCRPC. Methods. We retrospectively included $46 \mathrm{mCRPC}$ patients who received DOC followed by CBZ at quaternary hospitals in Japan between February 2015 and March 2019. Participants received intravenous DOC $\left(40-75 \mathrm{mg} / \mathrm{m}^{2}\right)$ every 3-4 weeks; CBZ $\left(15-25 \mathrm{mg} / \mathrm{m}^{2}\right)$ was administered every 3-4 weeks. Androgen-deprivation therapy and prednisolone $5 \mathrm{mg}$ (twice daily) were administered throughout both regimens. The primary endpoints were overall (OS) and progression-free survival (PFS). The secondary endpoints were the rates of $\geq 30 \%$ and $\geq 50 \%$ reduction in prostate-specific antigen (PSA) levels at chemotherapy initiation. Results. Participants were divided into two groups according to DOC cycles (Groups A and B: $\leq 6$ and $\geq 7$ DOC cycles, respectively). The rates of $\geq 30 \%$ and $\geq 50 \%$ reduction in PSA levels were higher in Group B than in Group A, but there were no significant differences in both groups. Median OS in Groups A and B was 12.7 and 71.0 months, respectively $(P<0.001)$; median PFS in Groups A and B was 3 and 12 months, respectively $(P<0.001)$. Conclusions. Administration of $\geq 7$ cycles of DOC followed by CBZ may improve oncological outcomes in patients with mCRPC.

\section{Introduction}

In Japan, prostate cancer is the fourth commonest cancer in men and the seventh cause of cancer mortality and has shown a rapid increase in prevalence in recent years [1]. Androgen-deprivation therapy (ADT) is a standard treatment option for advanced or metastatic prostate cancer (PCa) [2]. Approximately $80 \%$ of patients have decreased levels of prostate-specific antigen (PSA) and have been reported to experience reduction of primary or metastatic sites; however, most patients experience disease progression, especially metastatic castration-resistant $\mathrm{PCa}$ (mCRPC), within a median of 3 years after diagnosis [3].

The management of mCRPC has dramatically evolved in recent years. To date, in Japan, five agents are used for patients with mCRPC to improve oncological outcomes, including overall (OS) and progression-free survival (PFS). In 2004, docetaxel (DOC) became the first chemotherapeutic agent to be approved by the US Food and Drug Administration for first-line treatment of 
mCRPC [4]. Since 2014, 4 chemotherapeutic agents have been approved in Japan. Cabazitaxel (CBZ), a secondgeneration taxane, is approved for the treatment of patients with $\mathrm{MCRPC}$ who have previously received a DOCcontaining regimen [5]. Other therapies include androgen receptor-axis-targeted agents (ARATs), such as abiraterone acetate and enzalutamide, for $\mathrm{MCRPC}$ in the pre- and post-DOC settings [6-9], as well as radiopharmaceutical radium-223 for mCRPC with symptomatic bone metastases without visceral lesions [10].

The ARATs are recommended in the first-line treatment of asymptomatic patients with low-grade or low-volume mCRPC until radiological or symptomatic progression, whereas chemotherapy is recommended for symptomatic, high-metastatic burden or a rapid disease progression [11]. Among these agents, the combination DOC and prednisone therapy is the first regimen to have demonstrated an increase in survival and has become the standard-of-care in chemotherapy [4]. In a retrospective analysis of data from the European database, a sequence including DOC, CBZ, and ARATs provided the greatest benefit of OS in patients with mCRPC [12]. However, it is unclear whether switching to second-line chemotherapy or optimal sequencing of CBZ or ARATs leads to better clinical outcomes, because only few prospective randomized trials have been conducted on this therapeutic aspect [13].

This study was conducted to evaluate the efficacy of sequential therapy with DOC followed by CBZ and the impact of the number of prior DOC cycles on oncological outcomes in patients with mCRPC.

\section{Methods}

2.1. Patients. This study retrospectively included 46 patients with $\mathrm{mCRPC}$ who received DOC followed CBZ at quaternary hospitals in Japan between February 2015 and March 2019. All enrolled patients had histologically confirmed PCa with distant metastases. Clinicopathological and laboratory data included patient's age, height, weight, and serum PSA level at initial diagnosis of PCa and before the administration of CBZ, Gleason Grade Group, whether the patients underwent definitive therapy, number of cycles of DOC, use of ARATs, metastatic sites, lactate dehydrogenase (LDH), alkaline phosphatase (ALP), and hemoglobin (Hb). The Gleason Grade of the prostate biopsy cores was evaluated in compliance with the 2014 guidelines of the International Society of Urological Pathology [14].

The documents regarding study protocol and informed consent were approved by the Gifu University Institutional Review Board (approval no. 2019-210) and by each institutional review board.

2.2. Definition of CRPC. CRPC was defined per the Prostate Cancer Clinical Trials Working Group 3 criteria: $a \geq 25 \%$ increase in PSA levels, an absolute $\geq 2 \mathrm{ng} / \mathrm{mL}$ increase from the post-ADT nadir or radiographic progression in soft tissue or bone with or without PSA progression, and ongoing ADT with a serum testosterone level of less than $50 \mathrm{ng} / \mathrm{mL}$ [15]. Metastatic prostate cancer was defined as the presence of at least one metastatic lesion after staging using thoraco-abdominopelvic computed tomography and bone scintigraphy.

2.3. Chemotherapeutic Regimens. All the study participants received intravenous DOC $\left(40-75 \mathrm{mg} / \mathrm{m}^{2}\right)$ every 3-4 weeks [4]. CBZ was administered as a 3- or 4-weekly $\left(15-25 \mathrm{mg} / \mathrm{m}^{2}\right)$ regimen based on the schedule reported in a clinical trial [5]. The dose and schedule of DOC and CBZ were modified according to the patients' general condition or the severity of adverse events (AEs) in each patient. In both regimens, ADT was maintained, and prednisolone $5 \mathrm{mg}$ was administered twice daily throughout the study period. DOC and CBZ were continued until PSA or radiographic progression, severe AEs, or refusal of treatment by the patient.

2.4. Statistical Analysis. The primary endpoint was oncological outcomes, including OS and PFS. The secondary endpoints were the rates of $\geq 30 \%$ and $\geq 50 \%$ reduction in PSA levels from the baseline value recorded at the initiation of chemotherapy. Data were analyzed using JMP ${ }^{\circledR} 13$ (SAS Institute Inc., Cary, NC, USA). OS was defined as the time from the induction of chemotherapy to death from any cause. PFS was defined as the time from the initiation of DOC followed by CBZ to the appearance of local or regional disease/metastasis. In addition, PFS after the administration of CBZ after DOC was defined as the time from the initiation of CBZ to $a \geq 25 \%$ increase in PSA levels from the post-DOC nadir, radiological progression, development of symptoms due to cancer progression, or death. Categorical and continuous variables were compared using Fisher's exact test and Mann-Whitney $U$ test. Post-chemotherapy survival was analyzed using the Kaplan-Meier method. Survival according to subgroup classification was analyzed with the log-rank test. All $P$-values were 2 -sided, and the significance level was set at $P=0.05$.

\section{Results}

3.1. Patient Characteristics. We enrolled 46 patients in this study, and patient characteristics are listed in Table 1 . The study participants were divided into two groups by the number of cycles of DOC: Group A comprised patients who received $\leq 6$ cycles of DOC followed by CBZ, whereas Group $B$ included patients who received $\geq 7$ cycles of DOC followed by CBZ. Table 2 summarizes patients' characteristics by the number of cycles of DOC. The number of cycles of DOC in the Group B was significantly higher than in Group A $(P<0.001)$; however, the ALP in Group A was significantly higher than in Group B $(P=0.039)$. In this study, data on the performance status, the number of metastatic sites, and the tumor volume within the metastatic sites could not be collected. There was no significant between-group difference in the initial dosage of DOC and CBZ.

3.2. Oncological Outcomes. At the end of the follow-up period, 35 patients $(76.1 \%)$ had died of PCa. The median 
TABLE 1: Patient characteristics.

\begin{tabular}{lc}
\hline Covariates & 46 \\
Number & $72(67-75)$ \\
Age (years, median, IQR) & $23.0(21.0-24.8)$ \\
Body mass index $\left(\mathrm{kg} / \mathrm{m}^{2}\right.$, median, IQR) & $113.9(15.5-653.1)$ \\
Initial PSA (ng/mL, median, IQR) & $0(0)$ \\
\hline Gleason Grade (number, \%) & $43(93.5)$ \\
$\leq 2$ & $3(6.5)$ \\
$\geq 3$ & $11(23.9)$ \\
Unknown & $14.5(9.0-21.3)$ \\
Definitive treatment for prostate (number, \%) & $1(1-2)$ \\
Time to CRPC after initial diagnosis (months, median, IQR) & $8(4-16.5)$ \\
The number of the administration of ARTs (number, median, \%) & \\
The number of cycles of DOC (number, median, IQR) & $42(91.3)$ \\
Metastasis (number, \%) & $10(21.7)$ \\
Bone & $260(222-322)$ \\
Visceral & $297(243-524)$ \\
Lactate dehydrogenase (U/L, median, IQR) & $12.1(10.9-12.8)$ \\
Alkaline phosphatase (U/L, median, IQR) & \\
Hemoglobin (g/dL, median, IQR) & \\
\hline
\end{tabular}

$\mathrm{IQR}=$ interquartile range; $\mathrm{PSA}=$ prostate-specific antigen; $\mathrm{CRPC}=$ castration-resistant prostate cancer; $\mathrm{ART}=$ androgen receptor-axis-targeted agent; DOC $=$ docetaxel.

TABle 2: Patients' characteristics according to the number of cycles of docetaxel.

\begin{tabular}{|c|c|c|c|}
\hline & $\begin{array}{l}\text { Patients who administered } \leq 6 \text { cycles } \\
\text { of DOC }(N=21)\end{array}$ & $\begin{array}{l}\text { Patients who administered } \geq 7 \text { cycles } \\
\text { of DOC }(N=25)\end{array}$ & $P$ value \\
\hline Age (years, median, IQR) & $72(65-74)$ & $71(68-76)$ & 0.808 \\
\hline Body mass index $\left(\mathrm{kg} / \mathrm{m}^{2}\right.$, median, IQR) & $22.8(19.8-24.4)$ & $23.5(21.2-25.2)$ & 0.275 \\
\hline Initial PSA (ng/mL, median, IQR) & $61.2(8.5-358.0)$ & $159.0(27.0-825.6)$ & 0.209 \\
\hline Gleason grade (number, \%) & & & 0.585 \\
\hline$\leq 2$ & $0(0)$ & $0(0)$ & \\
\hline$\geq 3$ & $19(90.5)$ & $24(96)$ & \\
\hline Unknown & $2(9.5)$ & $1(4)$ & \\
\hline Definitive treatment for prostate (number, \%) & $5(23.8)$ & $6(24.0)$ & $>0.999$ \\
\hline $\begin{array}{l}\text { Time to CRPC after initial diagnosis (months, } \\
\text { median, IQR) }\end{array}$ & $11.0(5.5-22.0)$ & $15.0(13.0-21.5)$ & 0.064 \\
\hline $\begin{array}{l}\text { The number of the administration of ARTs } \\
\text { (number, median, \%) }\end{array}$ & $1(1-2)$ & $1(1-2)$ & 0.461 \\
\hline $\begin{array}{l}\text { Time from diagnosis of CRPC to DOC } \\
\text { administration (months, median, IQR) }\end{array}$ & $10.2(5.6-18.1)$ & $11.8(5.5-20.6)$ & 0.651 \\
\hline $\begin{array}{l}\text { The number of cycles of DOC (number, median, } \\
\text { IQR) }\end{array}$ & $4(3-5)$ & $16(9.5-28.5)$ & $<0.001$ \\
\hline DOC initial dosage $\left(\mathrm{mg} / \mathrm{m}^{2}\right.$, median, IQR) & $56(49-67.5)$ & $54(45-64.5)$ & 0.380 \\
\hline $\begin{array}{l}\text { The number of cycles of CBZ (number, median, } \\
\text { IQR) }\end{array}$ & $4(1.5-6)$ & $6(3-9.5)$ & 0.074 \\
\hline $\begin{array}{l}\text { CBZ initial dosage }\left(\mathrm{mg} / \mathrm{m}^{2}, \text { median, } \mathrm{IQR}\right) \\
\text { Metastasis (number, \%) }\end{array}$ & $20(18.8-20)$ & $20(20-20)$ & 0.926 \\
\hline Bone & $21(100)$ & $21(84.0)$ & 0.114 \\
\hline Visceral & $6(28.6)$ & $4(16.0)$ & 0.475 \\
\hline PSA at CBZ start (ng/mL, median, IQR) & $77.0(39.1-193.4)$ & $67.8(41.4-142.1)$ & 0.508 \\
\hline Lactate dehydrogenase (U/L, median, IQR) & $264(222-351)$ & $256(223-303)$ & 0.321 \\
\hline Alkaline phosphatase (U/L, median, IQR) & $331(277-527)$ & $255(197-495)$ & 0.038 \\
\hline Hemoglobin (g/dL, median, IQR) & $12.2(103-12.9)$ & $12.1(11.0-12.8)$ & 0.938 \\
\hline
\end{tabular}

$\mathrm{IQR}=$ interquartile range; $\mathrm{PSA}=$ prostate-specific antigen; $\mathrm{CRPC}=$ castration-resistant prostate cancer; $\mathrm{ART}=$ androgen receptor-axis-targeted agent; DOC $=$ docetaxel.

rates of $\geq 30 \%$ and $\geq 50 \%$ reduction in PSA levels after CBZ induction were $33.3 \%$ and $19.1 \%$, respectively. However, there was no significant differences in both groups $(P=0.742, P>0.999$, respectively; Figure 1$)$.
The median follow-up period for these patients was 9.0 months (interquartile range (IQR) 4.6-15.0 months). The median OS in all patients who were administered DOC followed by CBZ was 25.8 months and was 12.7 (95\% 


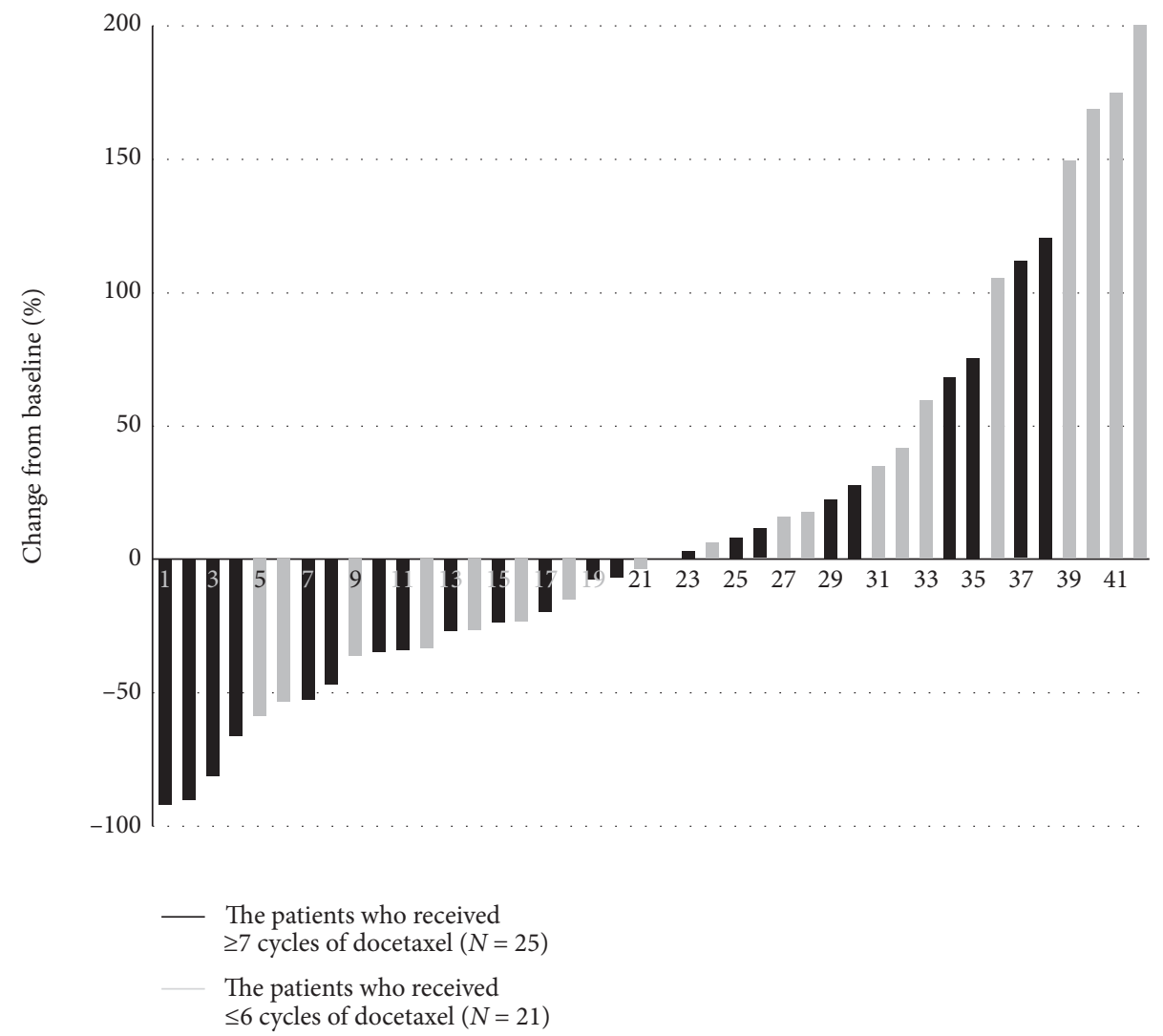

FIGURE 1: Waterfall plot of the maximum percentage change in prostate-specific antigen (PSA) levels from baseline. The median rates of $\geq 30 \%$ and $\geq 50 \%$ reduction in PSA levels after the induction of cabazitaxel in patients with metastatic castration-resistant prostate cancer who received $\geq 7$ cycles of docetaxel (DOC) followed by cabazitaxel (CBZ) were higher than those in patients who received $\leq 6$ cycles of DOC followed by CBZ, but there were no significant differences in both groups $(P=0.742$ and $P>0.999$, respectively).

confidence interval (95\% CI) $10.3-21.9)$ and 71.0 (95\% CI 32.5-90.1) months in Groups A and B, respectively $(P<0.001$; Figure 2). The median PFS in all patients who received DOC followed by CBZ was 6 months. The median PFS in groups A and B was 3 months (95\% CI 2.3-3.8) and 12 months (95\% CI 8.3-15.8), respectively $(P<0.001$; Figure 3). Moreover, the median OS after the induction of CBZ for all patients was 12.2 (95\% CI 7.7-14.5) months. The median OS in Group B was significantly longer than in Group A (16.4 months vs 7.7 months, respectively; $P=0.007)$. The median PFS at the induction of CBZ for all patients was 0.8 (95\% CI 0-3.0) months. Furthermore, the median PFS in the Group B was significantly longer than in Group A (2.1 months vs 0.4 months, $P=0.007$; Figure 4), respectively.

\section{Discussion}

Recent studies have shown that the administration of ARATs before chemotherapy improves OS [7,9]; therefore, there has been a therapeutic shift toward the use of ARATs as first-line treatment in patients with CRPC who have no or low-volume metastases [16]. Many patients have achieved remission owing to the clinical effectiveness of ARATs, but several patients have experienced rapid progression [17]. Patients with disease progression receive DOC in combination with prednisone as second-line treatment. However, the optimal sequence of treatment after DOC remains unclear because different treatment options are available at present, including CBZ [5], ARATs $[6,8]$, and radium-223 [10], without an adequate number of prospective randomized trials [13]. CBZ, a second-generation taxane, exhibits stronger suppression of microtubule dynamics, faster cellular uptake, and better intracellular retention than DOC and was selected for testing in clinical trials after it demonstrated an activity in DOC-resistant cell lines [18]. A recent prospective randomized trial conducted by CARD investigators showed that the proportions of imaging-based progression/death were $73.6 \%$ and $80.2 \%$ in patients with mCRPC who received CBZ and ARATs $(P<0.001)$, respectively, at a median follow-up of 9.2 months. [19]. In addition, the median OS and PFS were significantly longer in patients with MCRPC who received CBZ than in patients who received ARATs $(P=0.008$ and $P<0.001$, respectively) [19]. Angelergues et al. retrospectively investigated 574 consecutive mCRPC patients at 44 centers in six countries [12]. The patients were assigned to three groups according to the sequence of treatments: Group 1 was treated with the sequence $\mathrm{ADT} \longrightarrow \mathrm{DOC} \longrightarrow \mathrm{CBZ}$ only; Group 2 received $\mathrm{ADT} \longrightarrow \mathrm{DOC} \longrightarrow \mathrm{ARAT} \longrightarrow \mathrm{CBZ}$; and Group 3 received $\mathrm{ADT} \longrightarrow \mathrm{DOC} \longrightarrow \mathrm{CBZ} \longrightarrow \mathrm{ARAT}$ [12]. The median OS from the diagnosis of mCRPC in Groups 1, 2, and 3 was 38.3, 44.5, and 53.9 months, respectively ( $P=0.012$ for Group 3 vs. 


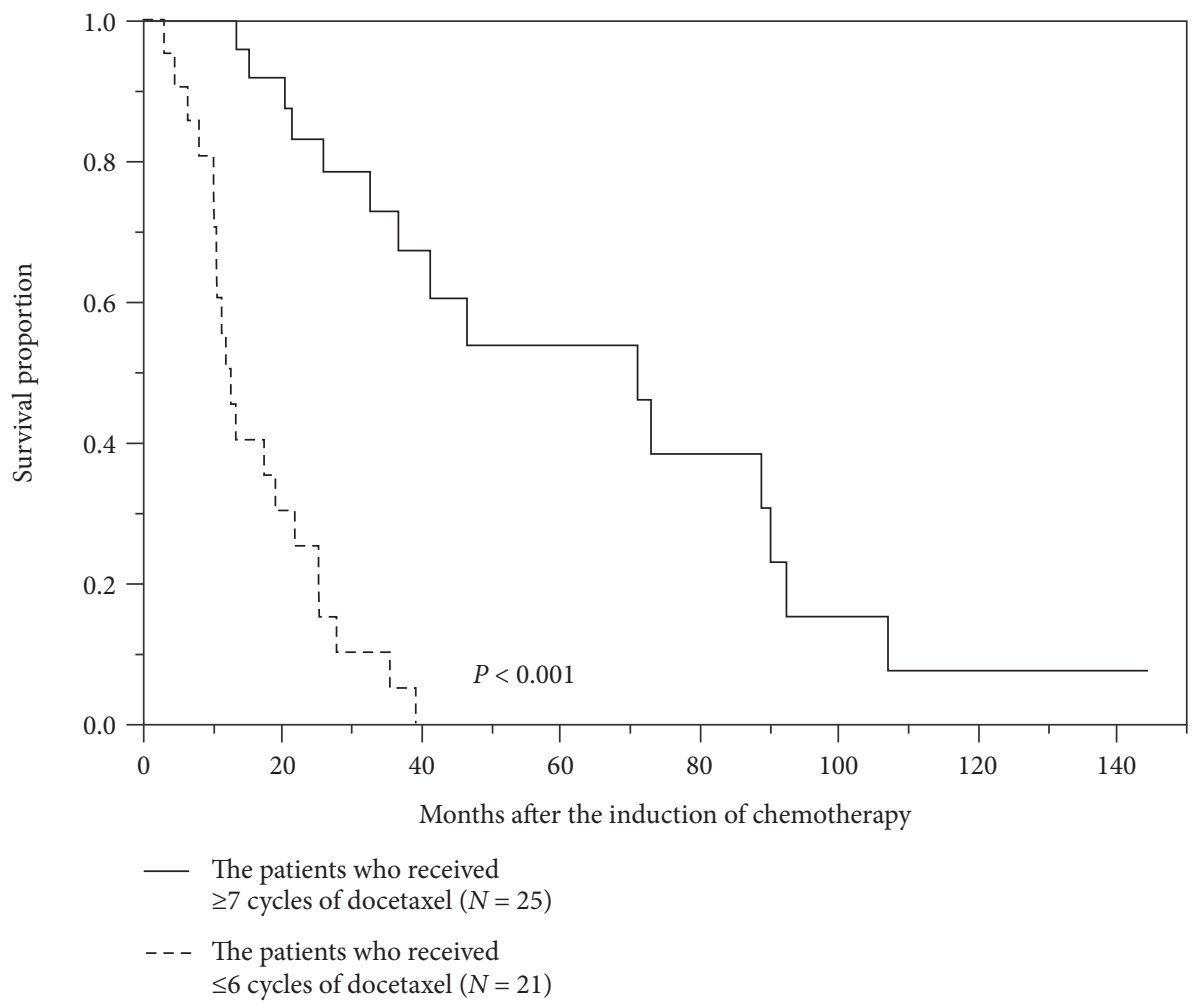

FIGURE 2: Kaplan-Meier estimate of overall survival (OS). The median OS in prostate cancer patients who received $\geq 7$ cycles of docetaxel (DOC) followed by cabazitaxel (CBZ) was significantly longer than that in patients who received $\leq 6$ cycles of DOC followed by CBZ $(P<0.001)$.

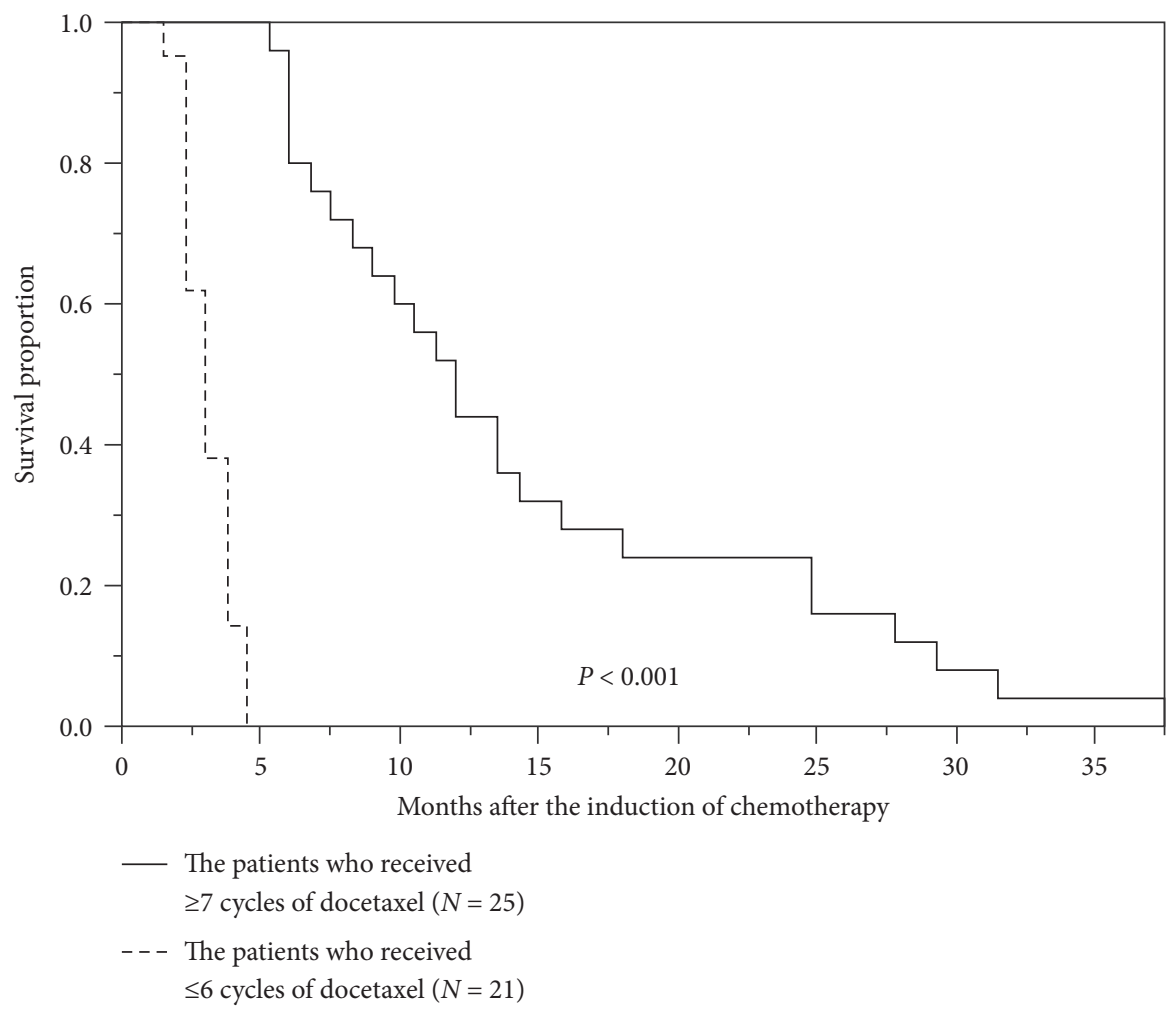

FIGURE 3: Kaplan-Meier estimate of progression-free survival (PFS). The median PFS in patients with prostate cancer who received $\geq 7$ cycles of docetaxel (DOC) followed by cabazitaxel (CBZ) was significantly longer than that in patients who received $\leq 6$ cycles of DOC followed by CBZ $(P<0.001)$ 


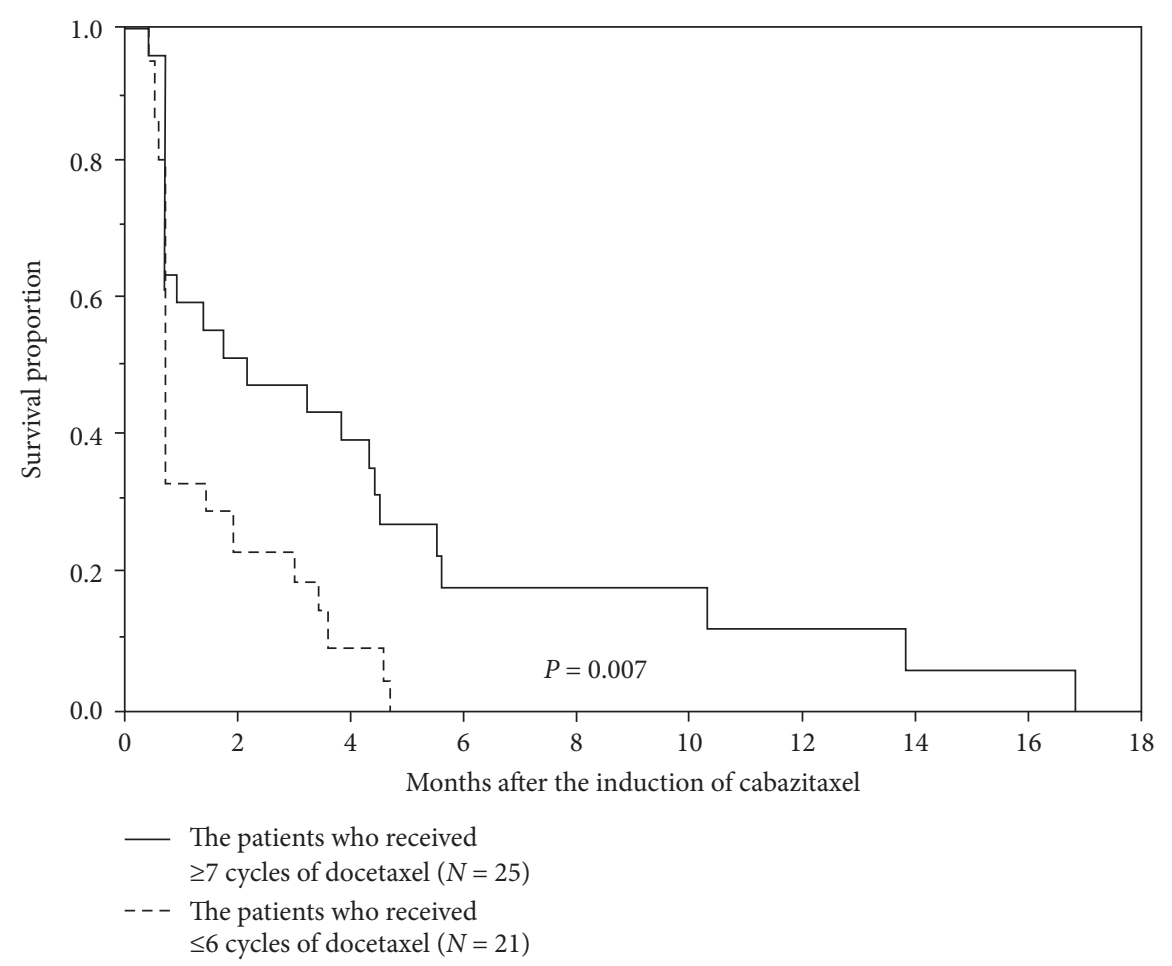

FIgURE 4: Kaplan-Meier estimate of progression-free survival (PFS) after the administration of cabazitaxel (CBZ). The median PFS in patients with prostate cancer who received $\geq 7$ cycles of docetaxel (DOC) followed by CBZ was significantly longer than that in patients who received $\leq 6$ cycles of DOC followed by CBZ $(P<0.007)$.

Group 1) [12]. Therefore, the researchers concluded that a sequence of DOC, CBZ, and ARAT therapy provided the most benefit in terms of OS [12]. Likewise, Oh et al. reported results from a retrospective observational study that evaluated whether taxane-based chemotherapy or ARAT as second-line treatment is associated improved oncological outcomes in patients with mCRPC who received ARATs as first-line therapy [17]. The PSA response rate was significantly higher in the chemotherapy cohort than in the ARAT cohort (40.9\% and $24.6 \%$, respectively; $P=0.005)$ [17]. Time to PSA progression was significantly longer in patients receiving chemotherapy after adjusting for covariates $(P=0.01)$ [17]. Therefore, sequential DOC followed by CBZ therapy may be beneficial in patients with $\mathrm{MCRPC}$ who have received ARATs as first-line therapy.

Several investigators have reported the efficacy of CBZ in patients with mCRPC treated with prior DOC $[5,12,17,19]$, but the optimal sequence for treatment with $\mathrm{CBZ}$ remains unclear in clinical practice. The clinical use of DOC chemotherapy has usually been limited to 10 cycles. Additionally, in a retrospective analysis of two clinical trials, a survival benefit was not detected in men with mCRPC who had undergone $>10$ cycles of DOC [20]. On the other hand, Shiota et al. reported that treatment-failure free and OS among patients with $\leq 10$ cycles of DOC were significantly shorter than among those with $>10$ cycles $(P=0.029$ and $P=0.039$, respectively), whereas there were no significant differences in PSA response and PFS [21]. Similarly, Tanaka et al. reported that the OS calculated from CRPC diagnosis or DOC induction was significantly longer in patients who received $\geq 11$ cycles of DOC than in patients who received $\leq 10$ cycles of DOC $(P=0.04$ and $P=0.04$, respectively) [22]. In the TROPIC trial, subgroup analysis showed excellent survival benefit in patients treated with $\geq 900 \mathrm{mg} / \mathrm{m}^{2}$ DOC [5]. Therefore, patients who received $>10$ cycles of DOC may obtain the persistent anticancer effects of $\mathrm{CBZ}$, despite $>10$ cycles of DOC being administered [21]. In contrast, Miyake and Kosaka reported that the response rate and PFS were not significantly different between DOC and $\mathrm{CBZ}[23,24]$. In the present study, $\geq 7$ sequential cycles of DOC followed by CBZ significantly improved the oncological outcomes in patients with mCRPC. Therefore, this result suggests that the total dose of DOC may be associated with prolonged OS and PFS in patients with CRPC who subsequently received $\mathrm{CBZ}$.

In Japan, the incidence of PCa has increased over the previous decades. Most Japanese patients with PCa received ADT as an initial treatment. For this reason, ADT is considered an effective treatment option for localized or locally advanced disease [25]. In addition, several studies reported that there were significant differences between Japanese and Western populations such as the characteristic manifestations of PCa or in terms of their tolerability to anticancer drugs [26]. Moreover, chemotherapy using DOC beyond 10 cycles has been approved for CRPC because it is covered by health insurance in Japan [25]. Therefore, the treatment strategy for mCRPC in Japan may be unique compared to other countries.

Several distinct prognostic factors were identified for the OS and PSA response of $\mathrm{mCRPC}$ patients treated with CBZ. Based on the TROPIC and SPARC trials, a prognostic model 
of OS after second-line chemotherapy in mCRPC patients treated with DOC was developed and externally validated using nine prognostic factors, including the Eastern Cooperative Oncology Group performance status (PS), time since last DOC use, measurable disease, presence of visceral disease, pain, duration of hormonal use, and levels of $\mathrm{Hb}$, PSA, and ALP [27]. In addition, Belderbos et al. identified that PS, Hb, ALP, and albumin (the World Health Organization) were significantly associated with OS [28]. However, it is difficult to obtain sufficient information about the primary tumor profile and castration-resistant metastatic sites. For these reasons, the biologic drivers of growth and cell-surface targets may change as the disease progresses through various treatments. Therefore, the Prostate Cancer Clinical Trials Working Group 3 recommends serial biologic profiling using tumor samples from biopsies or blood-based assays of circulating tumor cells (CTCs) or cell-free nucleic acids [15]. CTC enumeration and changes in CTC counts during treatment were identified as independent surrogate markers for survival outcomes of patients with mCRPC $[29,30]$. However, these biologic assessments are not available in general clinical practice in Japan. Therefore, a treatment tool is needed to select individualized therapies for individualized patients at individualized times [31].

4.1. Limitations. The present study has several limitations. First, it was a retrospective study and was conducted using multicenter data. Therefore, this study had an inherent potential for bias, with diagnostic and therapeutic variations among these institutions. Second, data on the performance status and tumor volume in the metastatic sites were not available in this study. In fact, serum ALP levels in Group A were significantly lower than those in Group B. Conversely, initial PSA levels in Group A were relatively lower and the time to castrate resistance after initial ADT was relatively shorter than those in Group B, although there were no significant differences in both groups. Furthermore, there were relatively more patients with bone and visceral metastases in Group A than in Group B. To this end, Group A may have more aggressive features and several poor prognostic factors, including the number of visceral metastases or the duration from the initiation of ADT to castration resistance, relative to Group B. Therefore, the administration of DOC in the patients in Group A may be discontinued because of PSA or radiographic progression. In addition, patients in Group B may achieve better oncological outcomes than those in Group A. Third, a relatively small number of patients were enrolled in this study. Therefore, a multivariate analysis could not be performed because of the small number of patients in each group. Furthermore, PSA doubling time and PSA velocity were not measured in this study. Finally, the rate of PSA reduction and overall response to DOC induction were not evaluated in this study.

\section{Conclusion}

The administration of $\geq 7$ cycles of DOC followed by CBZ may improve oncological outcomes, including OS and PFS, in patients with mCRPC. However, it may be necessary to switch from DOC to CBZ immediately for CRPC patients who had progressive disease, even if they administered DOC less than 7 cycles. Further large-scale clinical or case-control observational studies are needed to validate these findings.

\section{Abbreviations}

ADT: Androgen deprivation therapy

AE: Adverse event

ALP: Alkaline phosphatase

ARAT: Androgen receptor-axis-targeted agent

CBZ: Cabazitaxel

CI: Confidence interval

CRPC: Castration-resistant prostate cancer

CTC: Circulating tumor cell

DOC: Docetaxel

$\mathrm{Hb}$ : Hemoglobin

IQR: Interquartile range

LDH: Lactate dehydrogenase

OS: Overall survival

PCa: Prostate cancer

PFS: Progression-free survival

PSA: Prostate-specific antigen.

\section{Data Availability}

The data used to support the findings of this study are available from the corresponding author upon request. The study information was open for the public consumption at http://www.med.gifu-u.ac.jp//rinri/shinsa.html\#16.

\section{Ethical Approval}

All procedures performed in studies involving human participants were in accordance with the ethical standards of the institutional and/or national research committee and with the 1964 Helsinki declaration and its later amendments or comparable ethical standards.

\section{Conflicts of Interest}

The authors declare that there are no conflicts of interest regarding the publication of this paper.

\section{Authors' Contributions}

S. K., M. N., and T. K. were involved in the protocol/project development. M. T., K. I., S. F., M. N., and T. I. were responsible for the data collection or management. S. K., M. T., and M. N. analyzed the data. M. U., M. T., M. T., M. K., and Y. T. supervised the study. S. K. and T. K. wrote and edited the manuscript. All authors read and approved the final manuscript.

\section{References}

[1] H. Akaza, M. Onozawa, and S. Hinotsu, "Prostate cancer trends in Asia," World Journal of Urology, vol. 35, no. 6, pp. 859-865, 2017. 
[2] M. Shiota and M. Eto, "Current status of primary pharmacotherapy and future perspectives toward upfront therapy for metastatic hormone-sensitive prostate cancer," International Journal of Urology, vol. 23, no. 5, pp. 360-369, 2016.

[3] W. P. Harris, E. A. Mostaghel, P. S. Nelson, and B. Montgomery, "Androgen deprivation therapy: progress in understanding mechanisms of resistance and optimizing androgen depletion," Nature Clinical Practice Urology, vol. 6, no. 2, pp. 76-85, 2009.

[4] I. F. Tannock, R. de Wit, W. R. Berry et al., "Docetaxel plus prednisone or mitoxantrone plus prednisone for advanced prostate cancer," New England Journal of Medicine, vol. 351, no. 15, pp. 1502-1512, 2004.

[5] J. S. de Bono, S. Oudard, M. Ozguroglu et al., "Predonisone plus cabazitaxel or mitoxantrone for metastatic castrationresistant prostate cancer progressing after docetaxel treatment: a randomised open-label trial," Lancet, vol. 376, no. 9794, pp. 1147-1154, 2010.

[6] K. Fizazi, H. I. Scher, A. Molina et al., "Abiraterone acetate for treatment of metastatic castration-resistant prostate cancer: final overall survival analysis of the COU-AA-301 randomised, double-blind, placebo-controlled phase 3 study," The Lancet Oncology, vol. 13, no. 10, pp. 983-992, 2012.

[7] D. E. Rathkopf, M. R. Smith, J. S. De Bono et al., "Updated interim efficacy analysis and long-term safety of abiraterone acetate in metastatic castration-resistant prostate cancer patients without prior chemotherapy (COU-AA-302)," European Urology, vol. 66, no. 5, pp. 815-825, 2014.

[8] H. I. Scher, K. Fizazi, F. Saad et al., "Increased survival with enzalutamide in prostate cancer after chemotherapy," New England Journal of Medicine, vol. 367, no. 13, pp. 1187-1197, 2012.

[9] T. M. Beer, A. J. Armstrong, D. E. Rathkopf et al., "Enzalutamide in metastatic prostate cancer before chemotherapy," New England Journal of Medicine, vol. 371, no. 5, pp. 424-433, 2014.

[10] C. Parker, S. Nilsson, D. Heinrich et al., "Alpha emitter radium223 and survival in metastatic prostate cancer," New England Journal of Medicine, vol. 369, no. 3, pp. 213-223, 2013.

[11] S. Damodaran, J. M. Lang, and D. F. Jarrard, "Targeting metastatic hormone sensitive prostate cancer: chemohormonal therapy and new combinatorial approaches," Journal of Urology, vol. 201, no. 5, pp. 876-885, 2019.

[12] A. Angelergues, E. Efstathiou, R. Gyftaki et al., "Results of the FLAC European Database of metastatic castration-resistant prostate cancer patients treated with docetaxel, cabazitaxel, and androgen receptor-targeted agents," Clinical Genitourinary Cancer, vol. 16, no. 4, pp. e777-e784, 2018.

[13] R. J. Jones, "Sequencing systemic therapies in advanced prostate cancer: spoiled for choice but not for evidence," European Urology, vol. 66, no. 3, pp. 466-467, 2014.

[14] J. I. Epstein, M. B. Amin, V. E. Reuter, and P. A. Humphrey, "Contemporary Gleason grading of prostatic carcinoma," The American Journal of Surgical Pathology, vol. 41, no. 4, pp. e1-e7, 2017.

[15] H. I. Scher, M. J. Morris, W. M. Stadler et al., "Trial design and objectives for castration-resistant prostate cancer: updated recommendations from the prostate cancer clinical trials working group 3," Journal of Clinical Oncology, vol. 34, no. 12, pp. 1402-1418, 2016.

[16] P. Cornford, J. Bellmunt, M. Bolla et al., "EAU-ESTRO-SIOG guidelines on prostate cancer. Part II: treatment of relapsing, metastatic, and castration-resistant prostate cancer," European Urology, vol. 71, no. 4, pp. 630-642, 2017.
[17] W. K. Oh, W. Y. Cheng, R. Miao et al., "Real-world outcomes in patients with metastatic castration-resistant prostate cancer receiving second-line chemotherapy versus an alternative androgen receptor-targeted agent (ARTA) following early progression on a first-line ARTA in a US community oncology setting," Urologic Oncology, vol. 36, no. 11, pp. 500.e1-500.e9, 2018.

[18] P. Vrignaud, D. Sémiond, P. Lejeune et al., "Preclinical antitumor activity of cabazitaxel, a semisynthetic taxane active in taxane-resistant tumors," Clinical Cancer Research, vol. 19, no. 11, pp. 2973-2983, 2013.

[19] R. De Wit, J. De Bono, C. N. Sternberg et al., "Cabazitaxel versus abiraterone or enzalutamide in metastatic prostate cancer," New England Journal of Medicine, vol. 381, no. 26, pp. 2506-2518, 2019.

[20] G. R. Pond, A. J. Armstrong, B. A. Wood et al., "Evaluating the value of number of cycles of docetaxel and prednisone in men with metastatic castration-resistant prostate cancer," European Urology, vol. 61, no. 2, pp. 363-369, 2012.

[21] M. Shiota, M. Nakamura, A. Yokomizo et al., "Efficacy and safety of cabazitaxel for castration-resistant prostate cancer in patients with $>10$ cycles of docetaxel chemotherapy: a multiinstitutional study," Medical Oncology, vol. 36, no. 4, p. 32, 2019.

[22] M. Tanaka, T. Kimura, Y. Iwamura et al., "No survival benefit found after extended treatment with docetaxel for patients with castration-resistant prostate cancer," The Prostate, vol. 79, no. 14, pp. 1604-1610, 2019.

[23] H. Miyake, T. Sugiyama, R. Aki et al., "No significant impact of prior treatment profile with docetaxel on the efficacy of cabazitaxel in Japanese patients with metastatic castrationresistant prostate cancer," Medical Oncology, vol. 34, no. 8, p. 141, 2017.

[24] T. Kosaka, H. Hongo, K. Watanabe, R. Mizuno, E. Kikuchi, and M. Oya, "No significant impact of patient age and prior treatment profile with docetaxel on the efficacy of cabazitaxel in patient with castration-resistant prostate cancer," Cancer Chemotherapy and Pharmacology, vol. 82, no. 6, pp. 10611066, 2018.

[25] M. Onozawa, S. Hinotsu, T. Tsukamoto et al., "Recent trends in the initial therapy for newly diagnosed prostate cancer in Japan," Japanese Journal of Clinical Oncology, vol. 44, no. 10, pp. 969-981, 2014.

[26] H. Miyake, I. Sakai, K.-I. Harada, M. Muramaki, and M. Fujisawa, "Significance of docetaxel-based chemotherapy as treatment for metastatic castration-resistant prostate cancer in Japanese men over 75 years old," International Urology and Nephrology, vol. 44, no. 6, pp. 1697-1703, 2012.

[27] S. Halabi, C.-Y. Lin, E. J. Small et al., "Prognostic model predicting metastatic castration-resistant prostate cancer survival in men treated with second-line chemotherapy," JNCI Journal of the National Cancer Institute, vol. 105, no. 22, pp. 1729-1737, 2013.

[28] B. P. S. Belderbos, R. De Wit, E. O.-D. Hoop et al., "Prognostic factors in men with metastatic castration-resistant prostate cancer treated with cabazitaxel," Oncotarget, vol. 8, no. 63, pp. 106468-106474, 2017.

[29] A. M. Sieuwerts, W. Onstenk, J. Kraan et al., "AR splice variants in circulating tumor cells of patients with castrationresistant prostate cancer: relation with outcome to cabazitaxel," Molecular Oncology, vol. 13, no. 8, pp. 1795-1807, 2019.

[30] I. E. De Kruijff, A. M. Sieuwerts, W. Onstenk et al., "Circulating tumor cell enumeration and characterization in 
metastatic castration-resistant prostate cancer patients treated with cabazitaxel," Cancers, vol. 11, no. 8, p. 1212, 2019.

[31] W. Onstenk, A. M. Sieuwerts, J. Kraan et al., "Efficacy of cabazitaxel in castration-resistant prostate cancer is independent of the presence of AR-V7 in circulating tumor cells," European Urology, vol. 68, no. 6, pp. 939-945, 2015. 